\title{
BMJ Open Can unsuccessful treatment in primary medical institutions influence patients' choice? A retrospective cluster sample study from China
}

Yadong Niu, ${ }^{1,2}$ Liang Zhang, ${ }^{1,2}$ Ting Ye, ${ }^{1,2}$ Yan Yan, ${ }^{1,2}$ Yan Zhang ${ }^{1,2}$

To cite: Niu Y, Zhang L, Ye T, et al. Can unsuccessful treatment in primary medical institutions influence patients' choice? A retrospective cluster sample study from China. BMJ Open 2019;9:e022304. doi:10.1136/ bmjopen-2018-022304

- Prepublication history for this paper is available online. To view these files, please visit the journal online (http://dx.doi. org/10.1136/bmjopen-2018022304).

Received 24 February 2018 Revised 29 0ctober 2018 Accepted 7 November 2018

A Check for updates

C Author(s) (or their employer(s)) 2019. Re-use permitted under CC BY-NC. No commercial re-use. See rights and permissions. Published by BMJ.

${ }^{1}$ School of Health and Medicine Management, Tongji Medical College, Huazhong University of Science and Technology, Wuhan, China

${ }^{2}$ Research Centre for Rural Health Service, Key Research Institute of Humanities \& Social Sciences of Hubei Provincial Department of Education,

Wuhan, China

Correspondence to

Dr Yan Zhang;

yanzhang@hust.edu.cn

\section{ABSTRACT}

Objective China has been attempting to control the patients' choice of high-level medical institutions through series measures of first point of contact at primary medical institutions, but the outcome is considered poor. We aim to analyse whether unsuccessful treatment in primary medical institutions can lead to the patients' choice of high-level medical institutions.

Design A retrospective cluster sample study.

Setting The study setting was in Macheng city, Hubei province.

Participants The respondents are township-county (TC) patients (patients who first went to township hospitals and then county hospitals within 30 days for the same disease) who experienced unsuccessful treatment in primary medical institutions. A total of $2090 \mathrm{TC}$ patients were screened out based on the New Rural Cooperative Medical System database in 2013.

Main outcome measures The choice of patients between township hospitals (primary medical institutions) and county hospitals was observed. We compared TC patients' ratio of choosing county hospitals (RoCC) before TC experience with after TC experience. Thereafter, we compared RoCC of TC patients and non-TC patients (patients who did not experience TC) based on coarsened exact matching.

Results The ratio of TC for outpatient in township hospitals is $0.68 \%$ and that of TC for inpatient in township hospitals is $3.37 \%$. RoCC for TC disease increased from $20.8 \%$ to $35.5 \%$ ( $p<0.001)$, RoCC for other disease increased from $35 \%$ to $37.3 \%(p=0.01)$. TC patients had significantly higher RoCC than non-TC patients $(p<0.001)$. Conclusions Patients' choice of high-level medical institutions is highly associated with the experience of unsuccessful treatment in primary medical institutions. Moreover, people likely select high-level medical institutions thereafter regardless of conditions in rural China. Unsuccessful treatment in primary medical institutions is inevitable for patients. Thus, additional measures should be considered in lowering the potential risks for patients when treatments fail.

Trial registration number ChiCTR-00R-14005563.

\section{BACKGROUND}

Health resources are known to be limited. Therefore, if people of different need
Strengths and limitations of this study

- This is the first study focusing on the influence of unsuccessful treatment experience on the patients' choice of medical institutions in China

- This cluster sample study was conducted in a county with 880 thousand residents based on the New Rural Cooperative Medical System database, which contained all the inpatient and outpatient records of rural population in the county.

- Patients' choice of medical institutions before and after unsuccessful treatment experience was selected out

- Only attainable variables were included into matching, thereby limiting the matching performance.

- Only 1-year data were included, and further track is needed to strengthen the conclusion.

can obtain their corresponding level of health resource, the health system would achieve maximum efficiency and equity. However, quite many patients tend to seek for higher level healthcare and choose higher level medical institution than necessary when they need medical help, particularly in places where people are free to select their healthcare provider. For example, bypassing local medical institutions and going to lager medical institutions ${ }^{1-3}$ or going to the emergency department for mild illness. ${ }^{4}$ Those health-seeking behaviours have crowded high-level medical institutions and weakened or bankrupted local medical institutions, ${ }^{56}$ and result in rapid increasing of medical expenditure. ${ }^{7-9}$

In the $1980 \mathrm{~s}$, China introduced market mechanisms into the health delivery system. People could go to any level of medical institutions on their own will and the primary medical institutions no longer played the role of health gatekeeper. The reform developed the health industry but also result in hidden problems. People have progressively preferred high-level medical 
institutions. ${ }^{10}{ }^{11}$ Inappropriate choice gradually emerged out of free choice. ${ }^{12-14}$ Bypassing behaviour caused great waste because the price of the same service in high-level medical institutions is much higher than that in primary medical institutions. Bypassing behaviour also caused resource concentration in high-level medical institutions, which promoted more bypassing behaviour and more waste. To contain the health cost and reorient patients' choice of medical institutions, the Chinese government implemented a new health reform in 2009, in which tiered healthcare delivery system and first point of contact at primary medical institutions were raised as key tasks for the first time. A series of measures are implemented, such as making discriminatory compensation (patients will acquire more compensation if they seek medical help from lower level medical institutions), ${ }^{15} 16$ building regional medical association to promote dual referral $^{17} 18$ and increasing government investment on primary medical institutions. ${ }^{14}{ }^{19}$ In 2015, Chinese government even published guideline for building tiered healthcare delivery system to reinforce the implementation of first point of contact at primary medical institutions. However, the result is poor. ${ }^{1420} 21$ According to China Statistics Yearbook of Sanitation, the inpatient/outpatient service in primary medical institutions accounted for $32.6 \% / 65.1 \%$ of total inpatient/outpatient service in 2009 , but decreased to $19.1 \% / 56.3 \%$ in $2016 .{ }^{22}$

Many studies have focused on understanding patients' choosing behaviour. Factors from both patients (age, income, etc) and providers (attitude, price, etc) were found to have an influence on the patients' choice of health provider. ${ }^{23-25}$ However, none of those studies can explain why measures of first point of contact at primary medical institutions China has been pushing nationally failed to reduce the patients' choice of high-level medical institutions.

It is inevitable that patients would experience unsuccessful treatment now and then, which means that the service provided did not solve patients' health problem or did not reach their expectations. The possibility of unsuccessful treatment is related to the service capacity of providers. The lower capacity providers have the higher possibility that patients would experience unsuccessful treatment.

To date, few studies have paid attention to the influence of unsuccessful treatment experience on the patients' choice. Our assumption is that unsuccessful treatment experience in primary medical institutions can influence the patient's choice for high-level medical institutions, thereby leading first point of contact at primary medical institutions into failure. Imagining that a patient needs medical help and goes to a primary medical institution, if the treatment is unsuccessful, he or she may not choose it as the first contact of care next time.

Patients who experienced unsuccessful treatment in primary medical institutions are identifiable because they will transfer thereafter to another medical institution, particularly to high-level medical institutions. The health system in rural area of China is a tertiary structure, in which village clinics, township hospitals and county hospitals play major roles in health delivery. A village usually has one clinic, a town has one township hospital and a county has three to four public county hospitals. The township hospitals and village clinics belong to primary medical institutions. ${ }^{19}$ Patients who initially went to township hospitals (primary medical institution) and then county hospitals for the same disease are called township-county patients (TC patients). ${ }^{26}$ Accordingly, they experienced unsuccessful treatment in township hospitals (primary medical institutions); otherwise, they would not go to the county hospital for further treatment. We aim to validate the assumption above by considering TC patients as study objects. Two questions we intend to answer are:

1. Will patients' choice of medical institutions change after the experience of TC?

2. Is there any difference between TC patients and nonTC patients in choice of medical institutions?

\section{MATERIALS AND METHODS \\ Sample}

This retrospective cluster sample study was carried out in Macheng city, which is a typical county in Hubei province, central China. The city has a large population (approximately 880000 ) and medium gross domestic product per capital ( $¥ 22758$ or US $\$ 3705$ ). There are 4 public county hospitals and 20 township hospitals in Macheng (2013), and all the rural residents are employed in New Rural Cooperative Medical System (NRCMS, an insurance for rural residents in China). Information related to health service utilisation (including outpatient service and inpatient service) is recorded in the NRCMS database because of reimbursement (People would get compensated when any cost was incurred in medical institutions and the reimbursement covers only part of the cost). All the TC patients of Macheng in 2013 were included into our study and they were selected out based on the NRCMS database of Macheng in 2013. We also included all the patients who did not experience TC (non-TC patients) into our study as controls.

There are mainly three types of TC patients. Type 1 patient initially used outpatient service in a township hospital and then outpatient service in a county hospital (OTOC patients). Type 2 patient initially used outpatient service in a township hospital and then inpatient service in a county hospital (OTIC patients). Type 3 patient initially used inpatient service in a township hospital and then inpatient service in a county hospital (ITIC patients). Patient may also use inpatient service in township hospital first and then outpatient service in county hospital. Nevertheless, if patients were not suggested admission by doctor of county hospitals right after the discharge from township hospitals, it was highly possible that the service provided by township hospitals worked well. Besides, this rarely happens in actual situations. 


\section{Data processing}

Selection of TC patients

First, we selected out the information needed from the database of 2013, including identifier (encrypted), disease, time of service utilisation and institution of service utilisation. Second, we selected out the medical records (including outpatient and inpatient records) in county hospitals and township hospitals and adjusted the disease code (eg, the disease code of chronic obstructive pulmonary disease was adjusted from J44.900 to J44).

Thereafter, we sorted the medical records in ascending order with the identifier, disease and time as keywords to ensure that medical records of the same person for the same disease were arranged together by time. Then, we calculated the time interval between adjacent two medical records of the same person for the same disease. If the former choice of institution was township hospital, the later choice of institution was county hospital, and the time interval was less than 30 days, the two medical records would be marked as TC, and the patient would be marked as TC patient. We adopted the 30 days criteria from the definition of readmission, which usually means readmission within 30 days. ${ }^{27}$ TC patients were divided into three categories according to the service type (outpatient or inpatient) of two medical records: OTOC patients, OTIC patients and ITIC patients. The patients who did not experience TC were marked as non-TC patients.

\section{Service utilisation for TC disease and other disease}

The disease patients had when experiencing TC was marked as their TC disease. If a patient experienced TC more than once, then the disease of the first TC was marked as his TC disease. We comprised identifier and disease code into a new identifier, relied on which the TC patients' medical records for TC disease and other disease (not TC disease) were obtained.

\section{Service utilisation before TC and after TC}

The time when patients experienced TC was marked as their TC time. If a patient experienced TC more than once, then the time of the first TC was marked as his TC time. The medical records of TC patients in 2013 were divided into four part relying on TC time and TC disease: before TC for TC disease, before TC for other disease, after TC for TC disease and after TC for other disease.

\section{Statistical method}

It is hard to track the choice of medical institutions for each of the patients. Therefore, we used ratio of choosing county hospitals (RoCC) to analyse the choice change of TC patients and comparison of choice between TC patients and non-TC patients. RoCC means the percentage county hospital takes up among all the choices of health seeking for a period of time. It reflects patients' tendency of choosing medical institutions. This ratio was calculated at population level. The calculation method of RoCG was shown below:

$$
\mathrm{RoCC}=\frac{C H}{C H+T H} \times 100 \%
$$

$$
\begin{gathered}
\text { RoCG before TC }=\frac{C H b}{C H b+T H b} \times 100 \% \\
\text { RoCC after TC }=\frac{C H a}{C H a+T H a} \times 100 \%
\end{gathered}
$$

Where ' $\mathrm{CH}$ ' refers to number of service utilisation in county hospitals for all the patients, and ' $\mathrm{TH}$ ' refers to number of service utilisation in township hospitals for all the patients. 'CHb' refers to number of service utilisation in county hospitals before TC for all the TC patients, and 'THb' refers to number of service utilisation in township hospitals before TC for all the TC patients. 'CHa' refers to number of service utilisation in county hospitals after TC for all the TC patients and 'THa' refers to number of service utilisation in township hospitals after TC for all the TC patients.

Coarsened exact matching (CEM) was used to eliminate interruption from other factors when comparing RoCG of TC patients and non-TC patients. CEM is a Monotonic Imbalance Bounding matching method for improving the estimation of causal effects by reducing imbalance in covariates between treated and control groups, and has been used in many studies. ${ }^{28}$ The benefits of CEM could be referred to Blackwell et al and Iacus et al. ${ }^{28}{ }^{29}$ To select a matched sample of TC patients and non-TC patients, we performed CEM across the following variables: sex, age, family size, driving time to centre county hospital, capacity of local township hospital, traffic condition. Matching variables were selected based on former studies, ${ }^{243031}$ and available information from the database. The capacity of township hospitals was evaluated based on weighted rank-sum ratio method (indicators included were human resource, financial resource, equipment, medical service, public health service and other service) and the study has been published. ${ }^{32}$ We performed CEM for each type of TC Patients.

$\chi^{2}$ test was used to compare the choice of medical institution before TC and after TC, the choice of medical institution for TC patients and non-TC patients. Values with $\mathrm{p}<0.05$ (two tailed) were considered statistically significant. We used Microsoft Excel 2013 for data processing, Stata V.12.0 for $\chi^{2}$ test and CEM.

\section{Patient and public involvement}

No patients or public were involved in this research.

Patient information was anonymised and de-identified before analysis.

\section{RESULT}

\section{Summary statistics of TC patients}

In 2013, a total of 185790 patients used outpatient service and 23763 patients used inpatient service in township hospitals. We selected out 2090 TC patients (including 546 OTOC patients, 725 OTIC patients and 801 ITIC patients). The ratio of TC for outpatient in township hospitals is $0.68 \%(1271 / 185790)$ and that for inpatient in township hospitals is $3.37 \%$ (801/23 763). Table 1 presents the summary the TC patient statistics. Generally, male TC patients $(54.6 \%)$ outnumbered female TC patients. 


\begin{tabular}{|c|c|c|c|c|}
\hline & $\begin{array}{l}\begin{array}{l}\text { OTOC } \\
(n=564)\end{array}\end{array}$ & $\begin{array}{l}\text { OTIC } \\
(n=725)\end{array}$ & $\begin{array}{l}\text { ITIC } \\
(n=801)\end{array}$ & $\begin{array}{l}\text { In total } \\
(n=2090)\end{array}$ \\
\hline \multicolumn{5}{|l|}{ Sex } \\
\hline Male & 56.9 & 64.1 & 44.3 & 54.6 \\
\hline Female & 43.1 & 35.9 & 55.7 & 45.4 \\
\hline \multicolumn{5}{|l|}{ Age } \\
\hline$<25$ & 41.0 & 15.3 & 18.2 & 23.3 \\
\hline $25-45$ & 22.5 & 31.0 & 7.9 & 19.9 \\
\hline $45-65$ & 27.7 & 34.6 & 40.0 & 34.8 \\
\hline$>65$ & 8.9 & 19.0 & 34.0 & 22.0 \\
\hline \multicolumn{5}{|c|}{ Family size $($ mean $\pm S D)$} \\
\hline & $4.4 \pm 1.7$ & $4.2 \pm 1.6$ & $4.0 \pm 1.7$ & $4.2 \pm 1.7$ \\
\hline \multicolumn{5}{|c|}{ Driving time to centre county hospital } \\
\hline$<15 \min$ & 7.8 & 3.9 & 4.0 & 5.0 \\
\hline $15-30 \mathrm{~min}$ & 14.4 & 9.8 & 12.0 & 11.9 \\
\hline $30-60 \mathrm{~min}$ & 64.2 & 66.8 & 60.5 & 63.7 \\
\hline$>60 \mathrm{~min}$ & 13.7 & 19.6 & 23.5 & 19.5 \\
\hline \multicolumn{5}{|c|}{ Capacity of local township hospital } \\
\hline High & 15.2 & 1.1 & 11.5 & 8.9 \\
\hline Medium & 50.9 & 69.7 & 62.3 & 61.8 \\
\hline Low & 33.9 & 29.2 & 26.2 & 29.3 \\
\hline \multicolumn{5}{|c|}{ Traffic condition } \\
\hline $\begin{array}{l}\text { National } \\
\text { highway }\end{array}$ & 55.5 & 45.8 & 43.4 & 47.5 \\
\hline $\begin{array}{l}\text { Provincial } \\
\text { highway }\end{array}$ & 25.7 & 20.6 & 24.0 & 23.3 \\
\hline $\begin{array}{l}\text { County } \\
\text { highway }\end{array}$ & 18.8 & 33.7 & 32.6 & 29.2 \\
\hline
\end{tabular}

ITIC, TC patient who used inpatient service in township hospital firstly, then inpatient service in county hospital within 30 days; OTIC, TC patient who used outpatient service in township hospital firstly, then inpatient service in county hospital within 30 days; OTOC, TC patient who used outpatient service in township hospital firstly, then outpatient service in county hospital within 30 days.

Most TC patients were over 45 years $(56.8 \%)$. TC patients had a family of 4.2 members in average. Most TC patients need to drive about 30-60 min to centre county hospital $(63.7 \%)$. Most TC patients lived with a township hospital of medium capacity and more TC patients lived near the national highway $(47.5 \%)$.

\section{Choice of hospitals for TC patients before and after TC Choice of hospitals before TC and after TC for TC disease}

The choice of hospitals before TC and after TC for TC disease is shown in figure 1. In general, hospital choice of TC patients changed after TC, and RoCC increased significantly $\left(\chi^{2}=131.105, \mathrm{p}<0.001\right)$. Hospital choice of different TC patients all changed after TC, and RoCC increased significantly (OTOC, $\chi^{2}=41.175, \quad \mathrm{p}<0.001$; OTIC, $\chi^{2}=35.893, \mathrm{p}<0.001$; ITIC, $\left.\chi^{2}=17.369, \mathrm{p}<0.001\right)$.

Choice of hospitals before TC and after TC for other disease (not TC disease)

The choice of hospitals before TC and after TC for other disease (not TC disease) is shown in figure 2. In general,

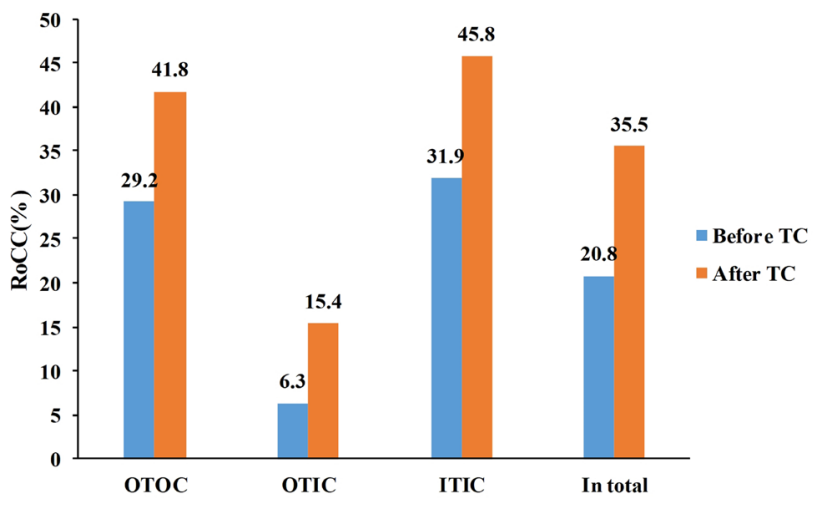

Figure 1 Hospital choice of TC patients for TC disease before and after TC in 2013. The sample size was shown in table 1. ITIC, TC patient who used inpatient service in township hospital firstly, then inpatient service in county hospital within 30 days; OTIC, TC patient who used outpatient service in township hospital firstly, then inpatient service in county hospital within 30 days; OTOC, TC patient who used outpatient service in township hospital firstly, then outpatient service in county hospital within 30 days; RoCC, ratio of choosing county hospitals; TC, township-county.

hospital choice of TC patients changed after TC, and RoCC increased significantly $\left(\chi^{2}=6.641, p=0.01\right)$. Hospital choice of different TC patients also changed after TC, RoCC of OTOC patients and OTIC patients increased significantly (OTOC, $\chi^{2}=5.324, p=0.021$; OTIC, $\chi^{2}=8.655$, $\mathrm{p}<0.05$ ), but RoCC of ITIC patients decreased significantly $\left(\chi^{2}=6.567, \mathrm{p}=0.01\right)$.

\section{Comparison of RoCC between TC patients and non-TC patients} CEM performance

The global imbalance measure L1 statistics before and after CEM are reported in table 2. The postmatching L1

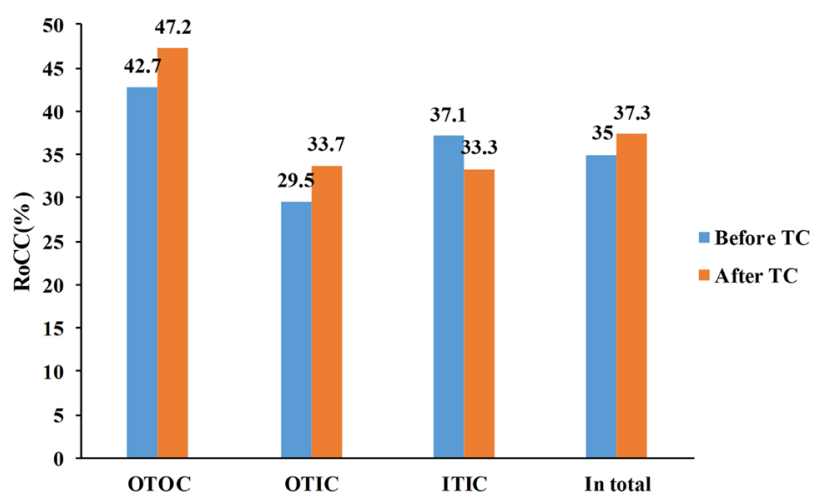

Figure 2 Hospital choice of TC patients for other disease before and after TC in 2013. The sample size was shown in table 1. ITIC, TC patient who used inpatient service in township hospital firstly, then inpatient service in county hospital within 30 days; OTIC, TC patient who used outpatient service in township hospital firstly, then inpatient service in county hospital within 30 days; OTOC, TC patient who used outpatient service in township hospital firstly, then outpatient service in county hospital within 30 days; RoCC, ratio of choosing county hospitals; TC, township-county. 


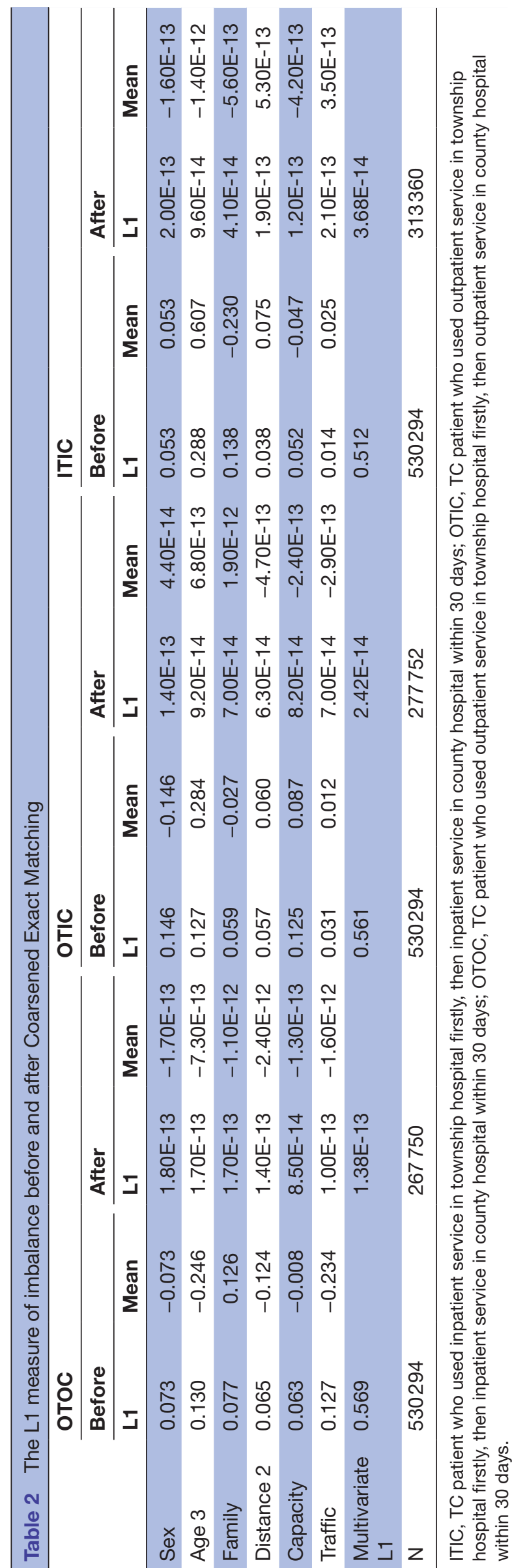

Table 3 Ratio of choosing county hospitals (RoCC) comparison of township-county (TC) patients and non-TC patients

\begin{tabular}{lllll}
\hline Patients & $\mathbf{n}$ & RoCC $\%$ & $\chi^{2}$ & P value \\
\hline OTOC & 563 & 42.3 & 469.587 & $<0.001$ \\
Non-OTOC & 267750 & 29.6 & & \\
OTIC & 725 & 30 & 123.809 & $<0.001$ \\
Non-OTIC & 277752 & 24.5 & & \\
ITIC & 800 & 39.2 & 614.387 & $<0.001$ \\
Non-ITIC & 313360 & 26.1 & & \\
\hline
\end{tabular}

ITIC, TC patient who used inpatient service in township hospital firstly, then inpatient service in county hospital within 30 days; OTIC, TC patient who used outpatient service in township hospital firstly, then inpatient service in county hospital within 30 days; OTOC, TC patient who used outpatient service in township hospital firstly, then outpatient service in county hospital within 30 days.

statistics are all close to 0 , as opposed to the prematching L1 statistics. The postmatching L1 statistics approximates 0 compared with the prematching L1 statistics. The final sample sizes for comparing RoCC between TC and non-TC patients are 267750 patients (including 563 OTOC patients), 277752 patients (including 725 OTIC patients) and 313360 patients (including 800 ITIC patients), respectively.

\section{RoCC of TC patients and non-TC patients}

We compared RoCC of TC patients and non-TC patients based on the result of CEM. As can be seen in table 3, all types of TC patients had significantly higher RoCC than non-TC patients.

\section{DISCUSSION}

\section{The ratio of TC in township hospitals was small}

The ratio of TC for outpatient service in township hospitals is considerably small (below 1\%). There are two possible reasons to explain this. One is that only a few patients would have unsuccessful treatment experience for outpatient service in primary medical institutions. The other is that patients chose not to attend the county hospital to avoid more cost, even the treatment in township hospital did not work. The ratio of TC for inpatient service in township hospitals is considerably higher than that for outpatient service $(3.37 \%)$. This result indicates that inpatients have a higher possibility of experiencing unsuccessful treatment than outpatients in primary medical institutions. The result makes sense because inpatients usually have more serious condition than outpatients.

\section{Patients likely choose county hospitals after TC}

We initially analysed the RoCC change of TC patients after TC and compared RoCC of TC patients and non-TC patients thereafter based on the CEM. The former results show that RoCC of TC patients for other disease increased after TC as well as for TC disease. The latter results show that RoCC of 
TC patients were significantly higher than that of non-TC patients. Besides, the sample size change of TC patients was little after CEM, therefore brought limited influence on the generalisability of conclusion. These findings indicate that the patients' choice afterwards is highly associated with TC or experience of unsuccessful treatment in primary medical institutions, and people are likely to choose county hospitals or higher level medical institutions when they are ill. Limited ability of township hospitals may promote choice for county hospitals. Patients would find that township hospitals lack ability in treating TC disease after the unsuccessful experience, so they went to county hospitals more. However, why they still prefer county hospitals for other disease? There are two possible reasons. First, dissatisfaction with township hospitals increases patients' bypassing behaviour. ${ }^{3633}$ The unsuccessful treatment in township hospitals bring more cost for patients on time and money (when they went to county hospitals directly, they would have saved the time and money they spent in township hospitals), thereby lowering the trust of patients on township hospitals and their willingness to choose township hospitals as the first contact of care. Second, insufficient cooperation owing to interest conflict between county hospitals and township hospitals drives choice for high-level medical institutions. County hospitals have been competing with township hospitals for patients and have no motivation to refer patients of mild condition (township hospitals are capable to handle) to township hospitals. If patients of mild condition can be referred to township hospitals, they may hold more trust on township hospitals. It is too subjective to say that all the choices of high-level medical institutions are inappropriate. However, when patients increasingly opt for high-level medical institutions regardless of conditions, there would be considerable inappropriateness among the choices.

\section{TC patients are not always prefer high-level medical institutions}

For ITIC patients, RoCC of TC disease increased after TC and RoCC of other disease decreased after TC, which was quite different from other types of TC patients. The decrease may be related to the disease burden. Hospital admission will bring immense financial burden for patients. Given that ITIC patients are admitted twice, OTOT patients are not admitted, OTIC patients are admitted once, ITIC patients have possibly heavier disease burden than OTOC and OTIC patients. ITIC patients need to avoid going to county hospitals as much as possible to save money for their family, thereby decreasing RoCC of other disease.

\section{Social changes strengthened the association}

TC or experience of unsuccessful treatment in primary medical institutions is inevitable and has existed before 1980s. Interestingly, why patients did not prefer highlevel medical institutions then? For one thing, the tertiary health system in rural areas allowed no free choice of health providers. That is, patients had to seek for medical help in sequence, first village clinics, then township hospitals, then county hospitals and lastly hospitals out of county. This situation occurs when low-level medical institutions cannot solve their health problems. For another, health resource was limited in the past, coverage of health insurance and household income were low, and patients hardly had the ability to go to high-level medical institutions. At present, patients in China have free choice of health providers, and they can go to any medical institution at will. Moreover, the increasing economic capacity of the Chinese residents and universal healthcare coverage further enhanced patients' ability to invest in health. The social changes above strengthened the association between choice of high-level medical institutions and unsuccessful treatment. Besides, increasing economic capacity also allows people to consume better non-technical medical service in higher level medical institution, which may also increase the tendency of bypassing.

Although the percentage of TC patients is small, the potential influence of unsuccessful treatment cannot be underestimated. Given that unsuccessful treatment may influence the choice of patients of medical institutions for any health problems, and they might also share this experience with their family, friends and neighbours, thereby exacerbating the consequences.

\section{Measures on lowering patients' potential risks at primary medical institutions are needed}

The Chinese government has implemented measures to promote first point of contact at primary medical institutions. Discriminatory compensation is the most relevant measure to patients, in which patients would have higher reimbursement rate if they go to lower level medical institutions. However, those measures remained ineffective. Prospect theory indicates that people are more sensitive to loss than gain. ${ }^{34}$ Patients cured through primary medical institutions can hardly realise that they have saved money (compared with the cost of going to large medical institutions). However, patients who think that their health problems are unsolved will feel that time and money are wasted. Therefore, economic measures, such as discriminatory compensation, are insufficient to lower patients' choice of medical institution. Moreover, the government must focus on avoiding or decreasing the additional cost for patients because of the low capability of primary medical institutions and lowering the potential risks of unsuccessful treatment when patients. Additional measures to promote cooperation and integrate care among different medical institutions are needed.

\section{Limitations}

Our study has several limitations. There are many other factors may influence the choice of medical institutions (income, disease severity and so on) ${ }^{24}{ }^{31}$ However, we did not include them into the CEM because the database did not have the information. The influence on choice of institutions may be an accumulated process, which means the more patients experience TC, the more significant the influence would be. However, we only studied the influence of 
the first TC in 1 year. Besides, whether the influence of TC can last over time remains to be further explored, and the TC influence found in 2013 may has been enhanced by TC before 2013 .

\section{CONCLUSION}

Our study suggests that patients' choice thereafter is highly associated with former experience of unsuccessful treatment in primary medical institutions and patients likely choose high-level medical institutions regardless of the disease. Although only a few patients would experience unsuccessful treatment in primary medical institutions, nobody wants to be one of them and considerable consequence will be caused when they ignore it. Additional measures should be considered to diminish unsuccessful treatments provided by primary medical institutions. Potential risks should also be reduced for patients when unsuccessful treatment happens.

Contributors NY and YZ participated in conception and design and the analyses, and wrote the manuscript. NY participated in data collection and performed the statistical analysis. TY, YY and LZ helped to review the manuscript and put forward modification opinions. YZ helped to draft the manuscript, review the manuscript and make final changes. All authors have given their final approval of the version to be published.

Funding This research is supported by the National youth Natural Science Foundation of China, grant number (71603088) and National Natural Science Foundation of China, grant number (71673099).

Competing interests None declared.

Patient consent for publication Not required.

Ethics approval The study protocol conformed to the guidelines of the Ethics Committee of the Tongji Medical College of Huazhong University of Science and Technology.

Provenance and peer review Not commissioned; externally peer reviewed.

Data sharing statement The anonymised dataset is available through the email of the corresponding author.

Open access This is an open access article distributed in accordance with the Creative Commons Attribution Non Commercial (CC BY-NC 4.0) license, which permits others to distribute, remix, adapt, build upon this work non-commercially, and license their derivative works on different terms, provided the original work is properly cited, appropriate credit is given, any changes made indicated, and the use is non-commercial. See: http://creativecommons.org/licenses/by-nc/4.0/.

\section{REFERENCES}

1. Mosadeghrad AM. Patient choice of a hospital: implications for health policy and management. Int $J$ Health Care Qual Assur 2014;27:152-64.

2. Yuqin M, Haiying T, Ning S, et al. Medical-care-seeking behavior of illness-caused poverty patients in rural areas and the influencing factors. Chinese General Practice 2016;19:100-5.

3. Sanders SR, Erickson LD, Call VR, et al. Rural health care bypass behavior: how community and spatial characteristics affect primary health care selection. J Rural Health 2015;31:146-56.

4. Durand AC, Gentile S, Devictor B, et al. ED patients: how nonurgent are they? Systematic review of the emergency medicine literature. Am J Emerg Med 2011;29:333-45.

5. Uscher-Pines L, Pines J, Kellermann A, et al. Emergency department visits for nonurgent conditions: systematic literature review. Am J Manag Care 2013;19:47-59.

6. Liu JJ, Bellamy GR, McCormick M. Patient bypass behavior and critical access hospitals: implications for patient retention. J Rural Health 2007;23:17-24.
7. Baker LC, Baker LS. Excess cost of emergency department visits for nonurgent care. Health Aff 1994;13:162-71.

8. Hsia RY, Friedman AB, Niedzwiecki M. Urgent care needs among nonurgent visits to the emergency department. JAMA Intern Med 2016;176:852.

9. McWilliams A, Tapp H, Barker J, et al. Cost analysis of the use of emergency departments for primary care services in Charlotte, North Carolina. N C Med J 2011;72:265-71.

10. Jinwei L. Health resource allocation between urban and rural of china: inverted triangle and causes. The World Of Survey And Research 2006:2228-24.

11. Jianhua F, Ping Z, Ping X, et al. Discuss on chinese medical resources allocation optimization: design for outpatient service space layout. Chinese Health Economics 2013;32:27-9.

12. Yue W, Shuang L, Liang Z. Analysis on vicious interaction mechanism of hierarchical medical institutions based on the relationship of resources and capacity. Chinese Health Economics 2017;36:66-9.

13. Lai W. Continuity - fragmentation-integration: Historical review of health service delivery modes from three-level health service networks in rural China. Chinese Journal of Health Policy 2014;7:24-30.

14. Shuguang $S$, Bo $Z$. Tiered healthcare delivery system, first point of contact at primary medical institutions and development of primary medical institutions. Academia Bimestris 2016;2:48-57.

15. Yizhao L, Wu W, Pang J, et al. Analysis of medieal insurance fund balance and study the effect of medieal insurance in hierarchical diagnosis and treatment. Chinese Primary Health Care 2016;30:12-13

16. Yihua $X$, Zhang $X$, Zhou $M$, et al. Analysis of related policy of differential payment of health insurance in China. Medicine and Society 2017;30:1-4.

17. Jeon SH. The long-term effects of cancer on employment and earnings. Health Econ 2017;26:671-84.

18. Yi L, Pei H, Shuqiu JI, et al. Analysis on the two-way referral by leading hospitals of three health alliances. Chinese Journal of Hospital Administration 2015;31:253-6.

19. Li X, Lu J, Hu S, et al. The primary health-care system in China. The Lancet 2017;390:2584-94.

20. Wang HH, Wang JJ, Wong SY, et al. The development of urban community health centres for strengthening primary care in China: a systematic literature review. Br Med Bull 2015;116:Idv043-53.

21. Wu D, Lam TP. Underuse of primary care in china: the scale, causes, and solutions. J Am Board Fam Med 2016;29:240-7.

22. National Health and Family Planning Commission of the People's Republic of China. 2017 Statistical Yearbook for health and family planning in China. Beijing: Peking Union Medical College press, 2017.

23. Glenngård $\mathrm{AH}$, Anell $\mathrm{A}$, Beckman $\mathrm{A}$. Choice of primary care provider: results from a population survey in three Swedish counties. Health Policy 2011;103:31-7.

24. Victoor A, Delnoij DM, Friele RD, et al. Determinants of patient choice of healthcare providers: a scoping review. BMC Health Serv Res 2012;12:272.

25. Qian D, Lucas H, Chen J, et al. Determinants of the use of different types of health care provider in urban China: a tracer illness study of URTI. Health Policy 2010;98:227-35.

26. Yadong $N$, Lei D, Shan L, et al. Validity analysis of readmissions at seven townships and counties of China. Chinese Journal of Hospital Administration 2016;32:618-21.

27. Stevens S. Preventing 30-day readmissions. Nurs Clin North Am 2015;50:123-37.

28. Blackwell M, lacus S, King G, et al. Cem: coarsened exact matching in stata. Stata J 2009;9:524-46.

29. lacus SM, King G, Porro G. Causal Inference without balance checking: coarsened exact matching. Political Analysis 2012;20:1-24.

30. Yip WC, Wang H, Liu Y. Determinants of patient choice of medical provider: a case study in rural China. Health Policy Plan 1998;13:311-22.

31. Brown PH, Theoharides C. Health-seeking behavior and hospital choice in China's New Cooperative Medical System. Health Econ 2009;18:S47-S64.

32. Jing J, Yan Z, Wenjun L, et al. Comprehensively evaluating the capacity of health service in township health centers of Macheng City based on weighted rank-sum ratio method. Chinese Health Service Management 2016;95:264-6.

33. Escarce JJ, Kapur K. Do patients bypass rural hospitals? Determinants of inpatient hospital choice in rural California. $J$ Health Care Poor Underserved 2009;20:625-44.

34. Tversky A, Kahneman D. The framing of decisions and the psychology of choice. Science 1981;211:453-8. 